\title{
Observation of Flaking Process in Rolling Contact Fatigue by Laminography Using Ultra-bright Synchrotron Radiation
}

\author{
Yoshikazu Nakai ${ }^{1,}$, , Daiki Shiozawa ${ }^{1}$, Shoichi Kikuch $^{2}$, Hitoshi Saito $^{1}$, Takashi Nishina ${ }^{1}$, \\ Taizo Makino ${ }^{3}$, and Yutaka Neishi $^{3}$ \\ ${ }^{1}$ Kobe University, Department of Mechanical Engineering, 1-1, Rokkodai, Nada, Kobe 657-8501, Japan \\ ${ }^{2}$ Shizuoka University, Department of Mechanical Engineering, 3-5-1, Johoku, Naka, Hamamatsu 432-8561, Japan \\ ${ }^{3}$ Nippon Steel \& Sumitomo Metal Corporation, Research \& Development, 1-8, Fuso, Amagasaki 660-0891, Japan
}

\begin{abstract}
The flaking failure in rolling contact fatigue (RCF) results from crack initiation and propagation has been believed to originate from non-metallic inclusions located beneath the surface. With conventional microscopies, however, damage process in the internal region of materials could not be observed, then RCF crack initiation and propagation behaviours were observed by using synchrotron radiation computed laminography (SRCL) in the brightest synchrotron facility in Japan, and the effect of the inclusion orientation on the RCF property was examined. In our previous studies, crack initiation and propagation behaviours caused by extended $\mathrm{MnS}$ inclusions distributed in depth or transverse (width) direction was observed by the SRCL. In the present study, the fracture mechanism under RCF was discussed on specimens with MnS inclusions distributed in the rolling direction. As a result, vertical cracks were initiated on the surface, parallel to the ball-rolling direction in specimens. The crack propagation direction was then changed perpendicular to the rolling direction. Thereafter, similar with our previous studies, vertical cracks caused the horizontal cracks beneath the surface, when the vertical cracks reached to a critical length. The ratio of the vertical crack initiation life to the flaking life was higher than specimens with other inclusion orientation.
\end{abstract}

\section{Introduction}

Roller bearings are used in various machinery such as cars, air planes and electrical appliances. In order to improve bearing life, it is necessary to investigate the rolling contact fatigue (RCF) mechanism. Bearings are generally applied under a pure rolling contact condition with oil lubrication without macroscopic slip, and RCF is a crucial factor for bearings. Since non-metallic inclusions are known to have a detrimental effect on the fatigue strength of high-strength steels, the effects of the composition (chemical/mechanical properties), shape, and orientation of inclusions on RCF should be clarified and controlled.

It has been recognized that flaking starts from inclusions beneath the surface where the ball track is located and the shear stress is greatest, and the cracks grow from them to the surface $[1,2]$. Martin et al. dealt with microstructural alterations, which develop with cyclic stressing under rolling contact, and attempted to define their nature and formation mechanisms [3]. In many cases, 'winglike' structural alterations, commonly called 'butterflies', were associated with the inclusions, as were microcracks [4-7]. Nagao et al., however, reported that the mechanism depended on the shape of the inclusions [8]. From spherical inclusions, shear-type cracks parallel to the rolling direction and surface were formed, while vertical cracks perpendicular to the rolling direction were first formed from stringer-shaped inclusions. In this case, shear-type cracks were formed after the formation of the vertical cracks. The formation and propagation of vertical cracks were considered to be controlled by the normal stress, while the propagation of horizontal cracks were controlled by the shear stress. Tsuchida and Tamura observed that horizontal cracks were formed from spherical inclusions, however, they considered that the crack initiation was controlled by the normal stress [9].

Since these phenomena occur beneath the surface, observations have been conducted destructively by cutting samples. Thus, successive observations of RCF have not been conducted. Synchrotron radiation micro computed tomography imaging (SRCT) has been applied for the non-destructive observation of the RCF process. Stiénon et al. calculated the stress field around nonmetallic inclusions in bearing steels in RCF tests using three dimensional (3D) shapes obtained by SRCT and at the European Synchrotron Radiation Facility (ESRF) [10,11]. Shiozawa et al. [12] and Makino et al. [13] used SRCT imaging to observe samples exhibiting flaking 


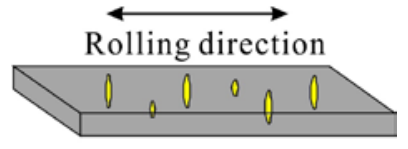

(a) S-Type sample

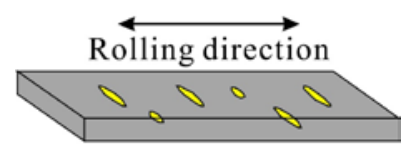

(b) T-Type sample

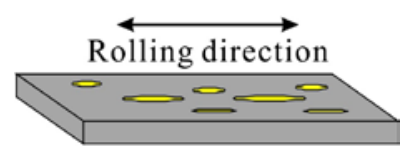

(c) L-Type sample

Figure 1. Orientation of inclusion.

damage and RCF-induced cracks. In their studies, samples were cut from normal-size RCF specimens so that they included damaged areas, and the 3D imaging of the damage before flaking provided useful information on the RCF crack initiation and propagation processes. To investigate the effect of the shape of inclusions on the crack initiation, artificial defects that simulate stringershaped inclusions were introduced into the specimens, and the crack initiation and propagation from the artificial defects were observed. For the successive SRCT imaging of the RCF process, samples must be sufficiently small to allow the transmission of X-rays, and the cross section must be smaller than $500 \mu \mathrm{m} \times 500$ $\mu \mathrm{m}$ for steels. Shiozawa et al. showed that the mechanism of RCF in a thin sample is different from that in a bulk sample [14]. Then, Nakai et al. [15] used synchrotron radiation computed laminography (SRCL) [16], which allows the high-resolution, non-destructive imaging of thin plates, to perform successive observations of the flaking process in RCF.

Nakai and others examined the effects of size and orientation of S-Type and T-Type samples those are designated in Figure 1 [17]. In most of previous RCF

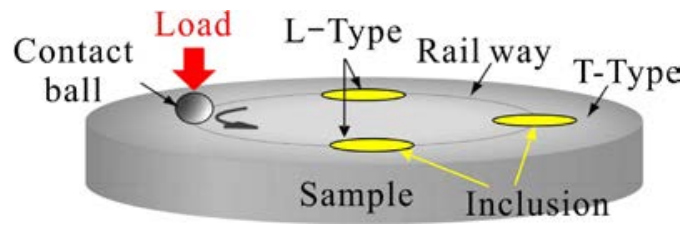

Figure 2. Thrust type rolling contact fatigue tester.

studies, thrust type machines with rotation of samples, shown in Figure 2, have been employed. Using this type machine, pure S-Type RCF test can be conducted. Pure T-Type or L-Type tests, however, cannot be performed because the rolling direction of each inclusion changes during the rotation of the sample. To accomplish pure T-Type RCF test, RCF tester with reciplocal motion has developed in our previous study $[14,15]$.

The flaking process in S-Type and T-Type samples is summarized in Figure 3 [17]. First a crack perpendicular to the rolling surface and the rolling direction forms from an inclusion that is adjacent to the rolling surface. Then the crack propagates vertically in the depth (thickness) direction. After the vertical crack propagates to a critical depth, a horizontal crack forms, which is parallel to the rolling surface. The horizontal crack propagates to form flaking.

In the present study, the RCF crack initiation and propagation in L-Type samples are examined on the basis of the observations obtained using SRCL.

\section{Experimental procedure}

\subsection{Material}

The material for the present study is a bearing steel (modified JIS SUJ2), whose chemical composition (in mass \%) is as follows: $1.00 \mathrm{C}, 0.35 \mathrm{Si}, 0.47 \mathrm{Mn}, 0.006 \mathrm{P}$, $0.017 \mathrm{~S}, 1.50 \mathrm{Cr}$, and balance $\mathrm{Fe}$. The material has intentionally contained high concentration of sulfur for the observation of the crack initiation from $\mathrm{MnS}$ inclusion. It was forged from an ingot with diameter of

(1) Vertical crack initiation (2) Vertical crack propagation (3) Horizontal crack formation (4) Horizontal crack from inclusion in the depth direction

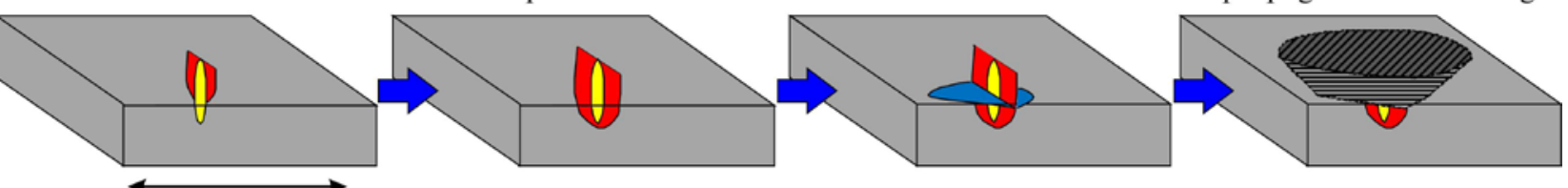

Rolling direction

(a) S-Type sample

(1) Vertical crack initiation (2) Horizontal crack formation (3) Vertical crack and from inclusion beneath the surface horizontal crack propagation

(4) Horizontal crack propagation and flaking

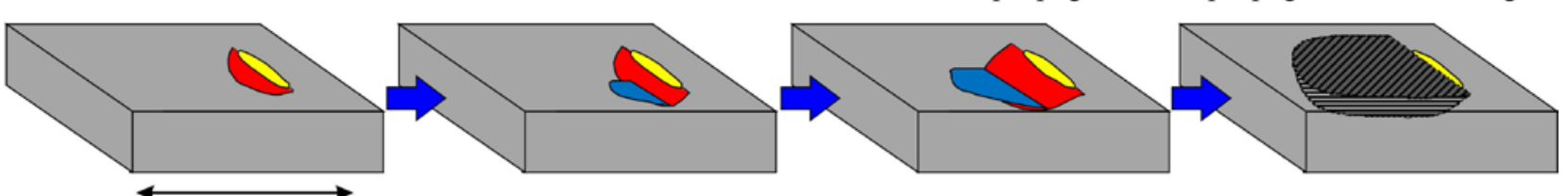

Rolling direction

(b) T-Type sample

Figure 3. Models of flaking mechanism for S-Type and T-Type samples. 


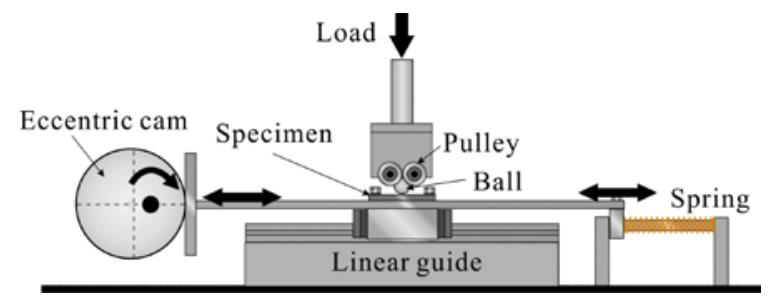

Figure 4. Schematic illustration of compact rolling fatigue testing machine.

$65 \mathrm{~mm}$, and inclusions are intergranular with a preferential alignment along the forging direction. After the spheroidizing annealing of cementite particles, specimens were cut from the forged bar, where the longitudinal direction of the bar corresponds to the rolling direction. The specimen was quenching at 1103 $\mathrm{K}$ for $0.5 \mathrm{~h}$ and tempered at $453 \mathrm{~K}$ for $2 \mathrm{~h}$. The dimension of the specimen for SRCL imaging was 10 $\mathrm{mm}$ in width, $24 \mathrm{~mm}$ in length and $1 \mathrm{~mm}$ in thickness. The thickness of the specimen was determined to allow transmitted X-ray with enough intensity for imaging.

\subsection{Rolling contact fatigue test}

To conduct RCF test for thin plate nearby the experimental hatch of beam lines of the synchrotron radiation facility, SPring-8, a special testing machine was developed. The schematic illustration of the new RCF testing machine is shown in Figure 4. The developed testing machine is boll-on-disk type contact tester. In this testing machine, reciprocal sliding motion is generated by a liner guide and an eccentric cam, and then the ceramic ball with diameter of $6.0 \mathrm{~mm}$ and Young's modulus of $300 \mathrm{GPa}$ rolls on the specimen linearly and reciprocally in napthenic lubricant oil with kinetic viscosity of $8.46 \mathrm{~mm}^{2} / \mathrm{s}$, unlike the thrust type rolling contact fatigue testing machine in which rolling is in a single direction, and the direction relative to the inclusions is changed during the rotation of specimen.

All RCF tests were conducted at the maximum Hertz stress of $5.39 \mathrm{GPa}$, where the sliding distance of this testing machine was $3.0 \mathrm{~mm}$. For observations of the crack initiation and propagation behaviours, fatigue tests were interrupted to conduct SRCL imaging. In this developed testing machine, a sample can be attached and removed easily.

\subsection{Laminography setup}

The measurement of SRCL imaging was carried out at BL46XU beam lines of Spring-8, which is the brightest synchrotron radiation facility in Japan. The method and apparatus of SRCL is shown in Figure 5. The axis inclination angle for the laminography, $\phi$, was $30^{\circ}$, and a monochromatic X-ray beam with $37 \mathrm{keV}$ was employed which was generated by undulator source. In the present study, the effective voxel size in the reconstructed 3D image was $0.74 \mu \mathrm{m}$. For 3D reconstruction, a set of 720 radiographs of a specimen were recorded over $360^{\circ}$ rotations, where each rotation

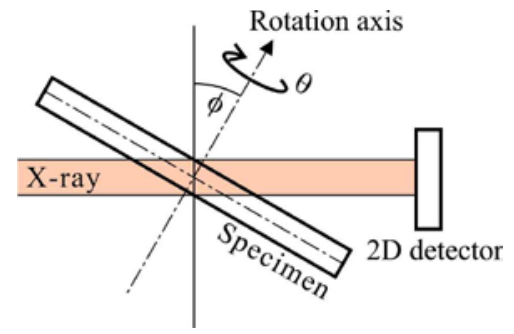

(a) Shematic illustration of SRCL imaging

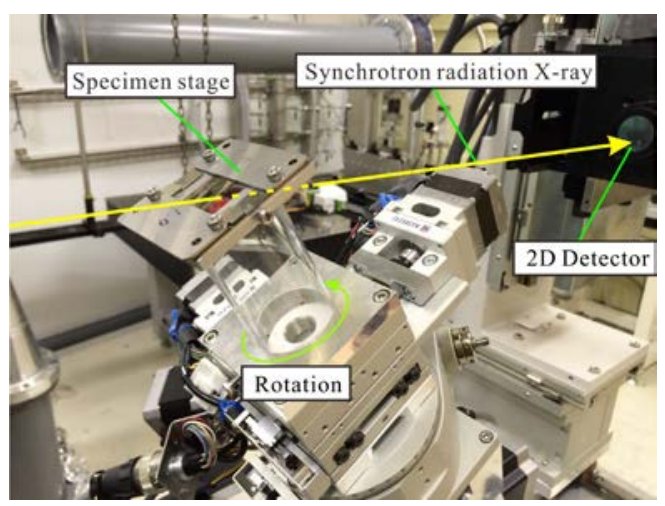

(b) SRCL imaging apparatus at SPring-8

Figure 5. SRCL imaging apparatus.

angle was $0.5^{\circ}$. The exposure time for each angle was 4 $\mathrm{s}$, and $1.5 \mathrm{~h}$ for one rotation including rotation of stage. To utilize the refraction contrast effect [18], the X-ray area detector was set by $0.35 \mathrm{~m}$ behind the sample. Once reconstructed, the 3D images have been visualized using software, Image-J and Amira. For 3D representation of inclusions and cracks, region growing method was employed to segment them [19], where gray value threshold between matrix and air was employed to produce binarized 3D images.

\section{Experimental results}

\subsection{Crack morphology at surface}

Figure 6 is representative micrographs showing the crack profiles at the surface those are observed by SEM. It is clear that cracks were formed from the tip of inclusions because of the stress concentration. The crack propagation direction depended on the orientation of inclusion. For S-Type sample and T-Type sample, the cracks propagate perpendicular to the rolling direction, and zigzag. It means that their propagations are controlled by the in-plane shear stress near the surface. For L-Type sample, however, cracks first propagated to the rolling direction, where the crack is almost straight. It means that the crack propagated by the tensile mode. After the crack length reached a length of $122 \mu \mathrm{m}$, the propagation direction was changes perpendicular to the rolling direction. The increase of stress intensity by crack extension may affect this change. After the change of the direction, the crack zigzag similar to S-type and Ttype inclusions.

For L-Type inclusion shown in Figure 6 (c), the flection of crack already occured at the first observation 


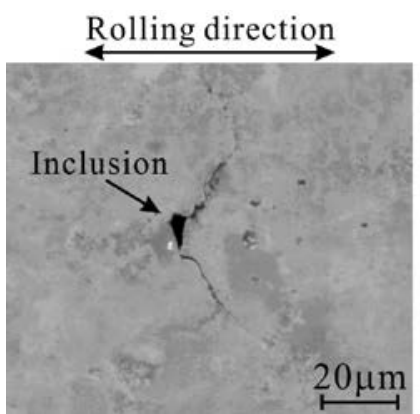

(a) S-Type

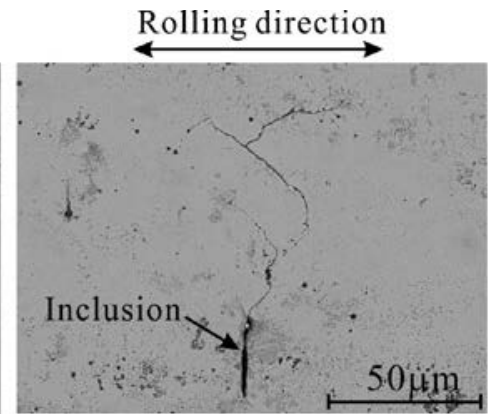

(b) T-Type

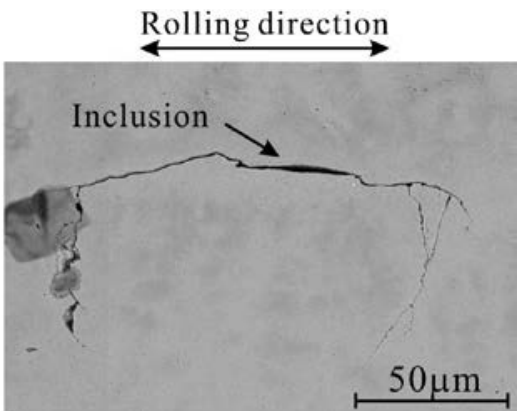

(c) L-Type

Figure 6. Crack initiation from inclusion and propagation at surface determined by replicas.

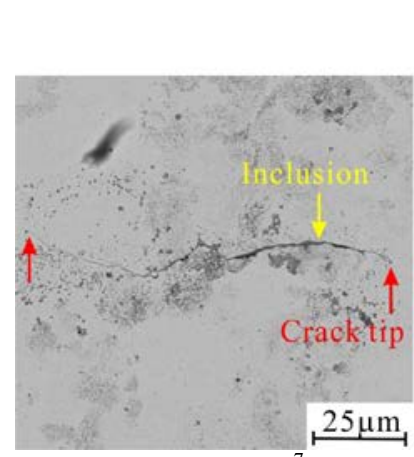

(a) $2.18 \times 10^{7}$ cycles

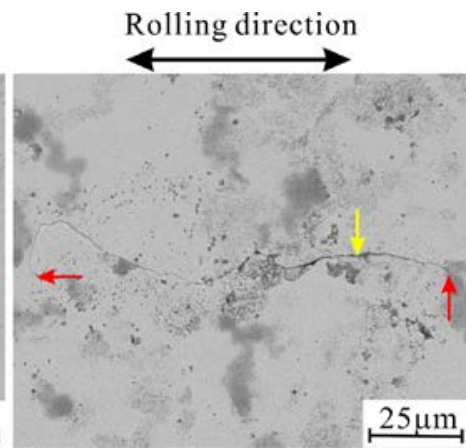

(b) $2.32 \times 10^{7}$ cycles

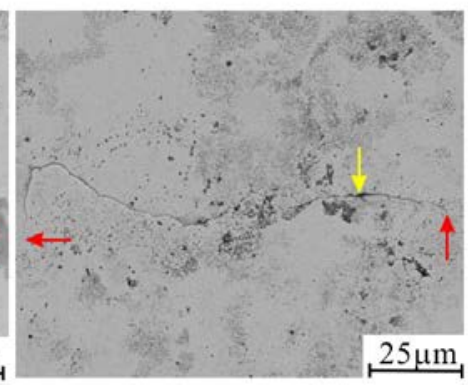

(c) $2.37 \times 10^{7}$ cycles

Figure 7. Crack initiation from inclusion and propagation at surface in L-Type sample determined by replicas..

after the start of RCF test, another observation was conducted, and the resuls are presented in Figure 7. In this observation, it is clear that the crack was initiated at the longitudinal tip of an inclusion, and propagated pararell to the rolling direction. After the crack reached a length of $113 \mu \mathrm{m}$, only the left side crack propagation changes perpendicular to the rolling direction between $2.18 \times 10^{7}$ and $2.32 \times 10^{7}$ cycles. The critical length of the crack propagation direction from longitudinal to the transverse direction is almost identical to that in Figure 5 (c).

\subsection{Laminography}

3D images of inclusions and cracks presented in Figure 6 (c) observed by SRCL at $N=1.50 \times 10^{7}$, $1.53 \times 10^{7}$, and $1.58 \times 10^{7}$ cycles, and shown in Figures 8 and 9 , where (A) shows top views, (B) shows views from the rolling direction, and $(\mathrm{C})$ shows side views of the specimen, and inclusions are indicated in pale yellow. Other color codes indicate the depth of the crack from the surface. As shown in the figures, the surface crack in Figure 6 (c) appears to form from a stringershaped inclusion with a length of about $30 \mu \mathrm{m}$ that adjacents to the surface, and the crack face is parallel to the rolling direction and vertical to the surface. At the first ofservation (a), however, both vertical and horizontal cracks were alrady formed, then it is not clear from these figure whether the formation of vertical crack or horizontal crack was first.

Then, the laminography of the crack shown in Figure 7 was conducted. The results are presented in Figure 10, where inclusions are indicated in pale yellow. Note that the other color code is different from that in Figures 8 and 9. Those indicate the number of cycles at each observation in this figure. In this case also a crack was first initiated from a tip of a longitudinal inclusion that located adjacent to the surface, and propagate to the rolling direction and vertical to the surface. After the longitudinal vertical crack propagated $113 \mu \mathrm{m}$, it changed perpendicular to the rolling direction between $2.18 \times 10^{7}$ and $2.32 \times 10^{7}$ cycles without forming a horizontal crack, and still vertical to the surface.

The flaking process of L-Type sample is summarized in Figure 11. (1) First, vertical cracks parallel to the rolling direction are formed and propagated until it reached a critical length. (2) Then, the vertical crack propagates perpendicular to the rolling direction. (3) When the vertical crack reached a critical depth, horizontal crack formed at the bottum of the vertical crack. (4) As a result of horizontal crack propagation, flaking is formed. Processes shown from (2) to (3) are similar to T-Type sample, while process (1) is an addition for L-Type sample.

\section{Discussion}

\subsection{Crack paths}

Figure 12 shows the potential mechanics for the difference of crack path between T-Type and L-Type samples. The stresses under contact with spherical ball are always axisymmetric. Because of the 
axisymmetry,crack can propagate to any directions, but
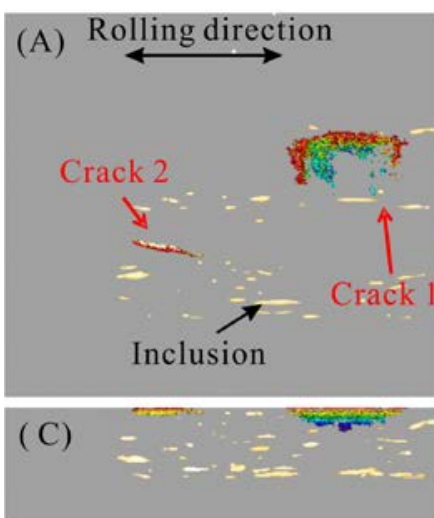

(a) $N=1.50 \times 10^{7}$ cycles
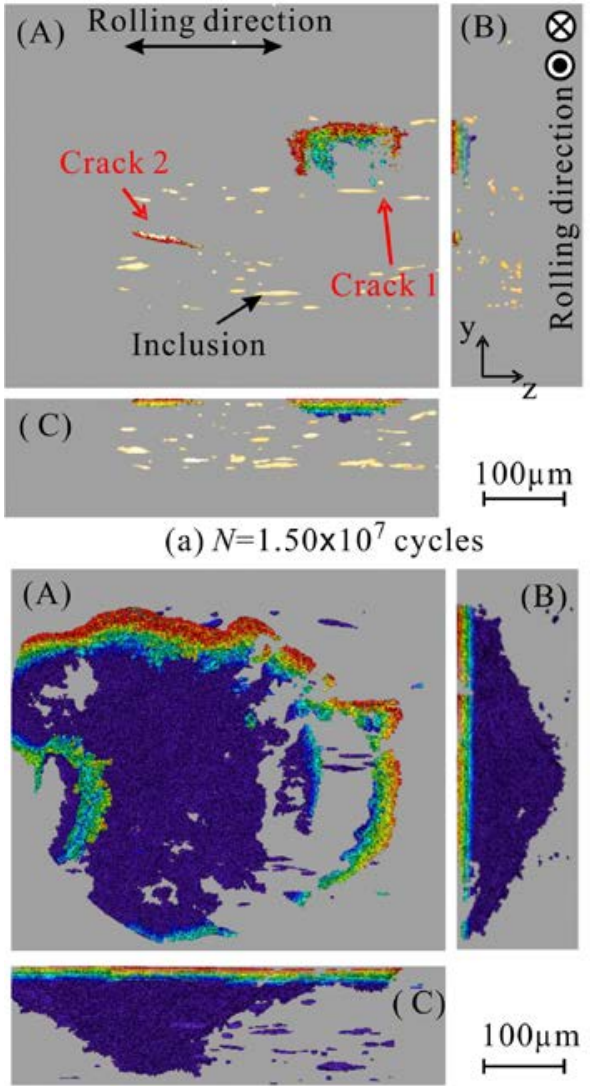

$100 \mu \mathrm{m}$

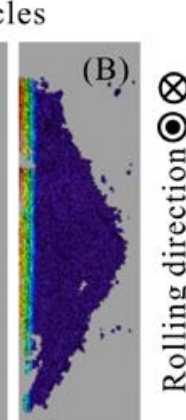

$100 \mu \mathrm{m}$ considering the movement of rolling ball, only the
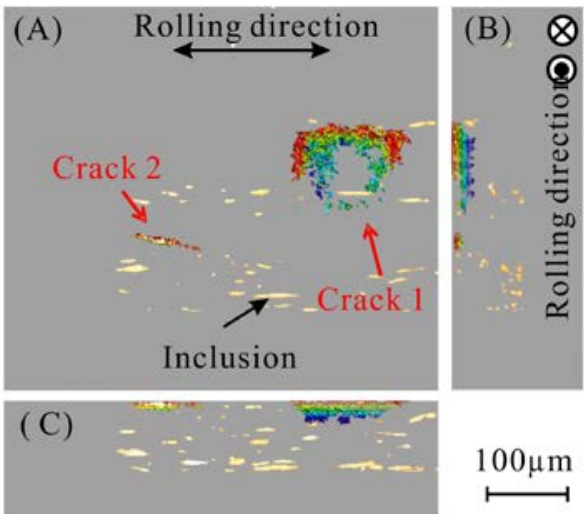

(b) $N=1.53 \times 10^{7}$ cycles

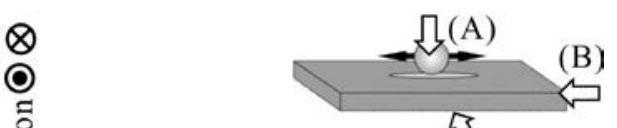

Depth $(\mu \mathrm{m})$

(C)

- $\begin{aligned} & 0 \sim 3 \\ & 3 \sim 6 \\ & \sim \sim 9\end{aligned}$

6 9

$9 \sim 12$
$12 \sim 15$

12 15

$15 \sim 18$

- $18 \sim 21$

- $21 \sim 24$

24

(c) $N=1.58 \times 10^{7}$ cycles

Figure 8. Laminographies showing flaking process for L-Type sample under $p_{\max }=5.39 \mathrm{GPa}$, where color code indicates depth of crack from the surface.

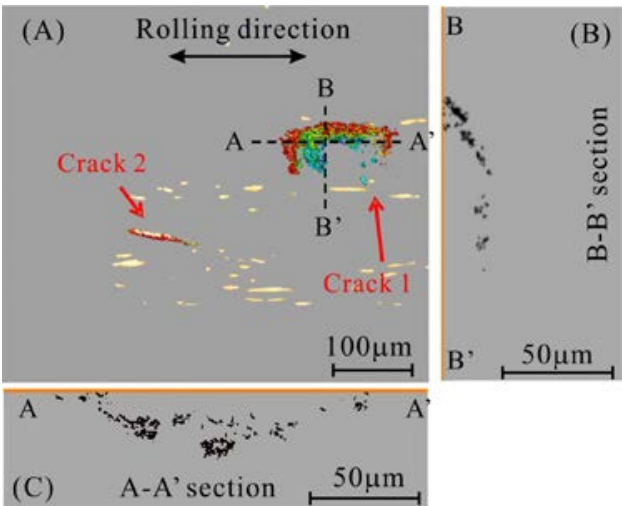

Figure 9. Crosssection of crack in Figure 7 (a) where color code is identical to that for Figure 8.

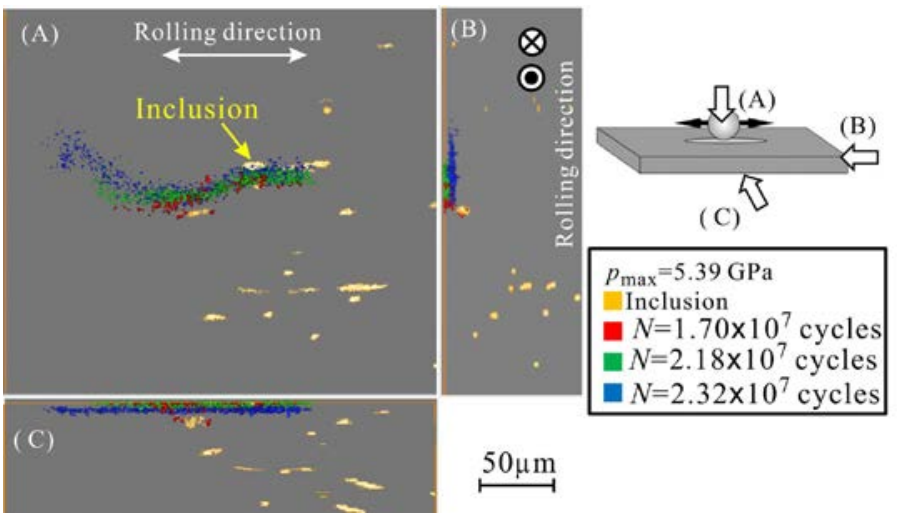

Figure 10. Laminography showing flaking process for L-Type sample, where color code indicates the number of cycles at each observation..

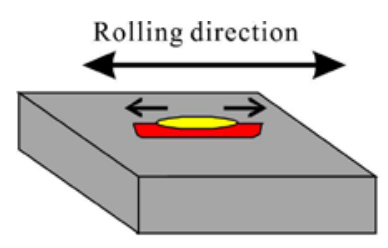

(1) Longitudinal vertical crack initiation and propagation

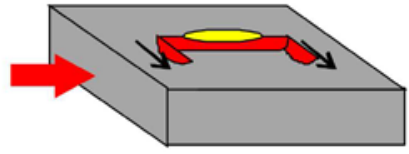

(2) Transverse vertical crack formation and propagation

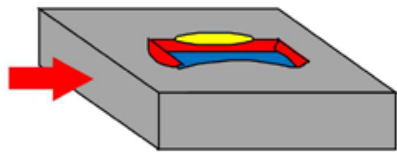

(3) Horizontal crack formation and propagation

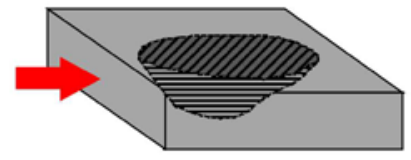

(4) Horizontal crack propagation and flaking

Figure 11. A model of flaking mechanism for L-Type sample. 


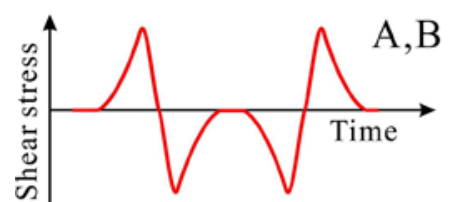

Shear stresses acting on planes perpendicular to rolling direction and parallel to surface

(a) Points A and B

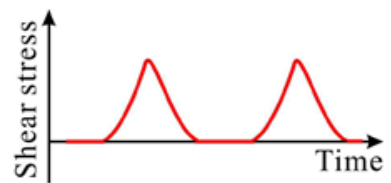

Shear stresses acting on planes parallel to rolling direction and parallel to surface

C

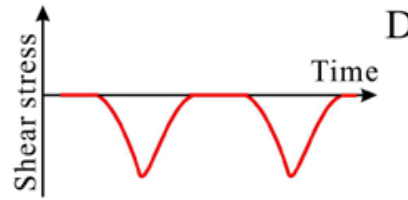

Shear stresses acting on planes parallel to rolling direction and parallel to surface

(b) Point C

(c) Point D

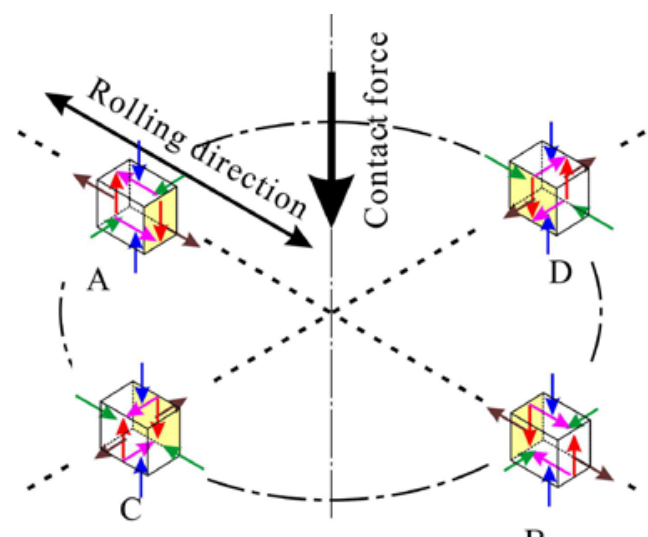

B

Figure 12. Axisymmetric stress field due to contact force.

change of the anti-plane shear stress can explain the difference of crack growth direction between S-Type, TType and L-Type samples because only the antiplane shear stress has different values in Cartesian coordinate system fixed to the sample. For S-Type and T-Type samples, the dominant stress component of the vertical crack propagation is acting on the plane perpendicular to the rolling direction. This shear stress changes alternately as shown in Figure 12 (a). Contrtary to them, the dominant stress component of the vertical crack propagation in L-Type sample is acting on the plane parallel to the rolling direction, and it is pulsating as shown in Figure 12 (b) and (c), where the stress

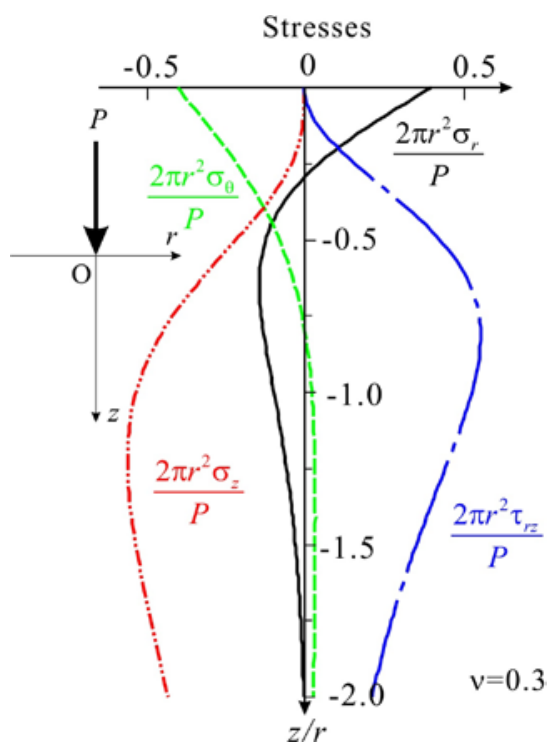

Figure 13. Stress distributio due to contact stress. amplitude is half for L-Type sample. Although L-Type sample has the same antiplane shear stress component at the transverse side as S-Type and T-Type sample, the stress concentration by inclusion [20] for the direction may not be enough for the crack initiation.

While the above discussion is valid for overall vertical crack path, the difference of crack morphology observed at the specimen surface cannot be explained. For more precise discussion, the stress components by the contact stress should be considered, those are given by the following equations and shown in Figure 13 [21].

$$
\begin{aligned}
& \frac{2 \pi r^{2} \sigma_{r}}{P(1-2 v)}=1-\frac{1}{\sqrt{1+(r / z)^{2}}}-\frac{3(r / z)^{4}}{\sqrt{\left[1+(r / z)^{2}\right]^{5}}} \\
& \frac{2 \pi r^{2} \sigma_{\theta}}{P(1-2 v)}=-1+\frac{1}{\sqrt{1+(r / z)^{2}}}+\frac{(r / z)^{2}}{\sqrt{\left[1+(r / z)^{2}\right]^{3}}} \\
& \frac{2 \pi r^{2} \sigma_{z}}{3 P}=-\frac{(r / z)^{2}}{\sqrt{\left[1+(r / z)^{2}\right]^{5}}} \\
& \frac{2 \pi r^{2} \tau_{r z}}{3 P}=\frac{(r / z)^{3}}{\sqrt{\left[1+(r / z)^{2}\right]^{5}}}
\end{aligned}
$$

This figure shows the stress distribution by concentrated contact point force, and the actual stress distribution by the distributed pressure induced by the ball contact should be obtained by integrating them, then the following discussion must be valid for the actual contact stress. 
Since the anti-plane shear stress indicated in Figure 13 is vanished at the specimen surface, the stress component which derive the crack initiation should be the radial stress, $\sigma_{r}$, and/or the circumferential stress, $\sigma_{\theta}$. Since $\sigma_{r}=-\sigma_{\theta}$, pure shear stress state is achieved at the specimen surface. Then the jigzag crack path induced by the shear stress is appeared for cracks whose face is perpendicular to the rolling direction. For L-Type sample, however, the path whose face is parallel to the rolling direction, is streight indicates that the crack path is controlled by the positive radial stress, $\sigma_{r}$.

Beneath the surface, the anti-plane shear stress componet controlls the crack path as shown in Figure 12, which has a conjugate components acting on the horizontal plane parallel to the specimen surface, while those two stress component have the same amplitude. A question arising here is which shear stress controlls the crack path. Just below the surface, this may be the stress actiong on the vertical plane because the normal stress on the plane is tension and the normal stress on the horizontal plane is compression. The compression stress leads the friction and reduces the driving force of the crack extension.

In deep site, however, this is the horizontal plane because the amplitude of the anti-plane shear stress reduces with the crack extension to the depth direction. On the other hand, the amplitude of the shear stress along a plane parallel to the surface keeps constant with crack extension to the longitudinal direction of the sample because of the horizontal movement of the contact point to the rolling direction.

The effect of the stress concentration induced by inclusions was not consided in the above discussion. Makino et. al. conducted the finite element analysis of the stress distribution for S-Type inclusion under the contact surface. Their analysis, however, the change of the stress component due to the movement of contact ball was not discussed.

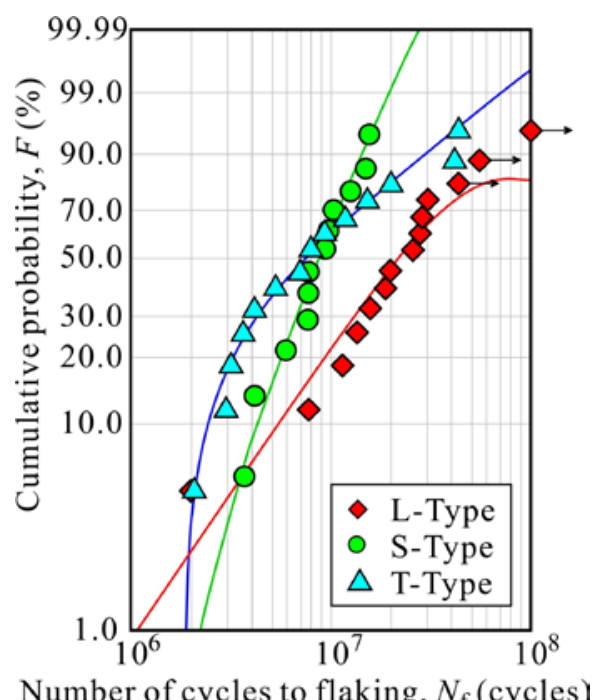

Figure 14. Rolling contact fatigue life.

\subsection{Fatigue life}

Weibull plot of the flaking life is shown in Figure 14. The flaking life at the probability of $50 \%$ is almost the same for S-Type and T-Type samples, but L-Type sample has much longer life.

To clarify which process defined in Figures 3 and 11 is responsible for the difference, the crack propagation life for each process is examined. Figure 15 shows the comparison of the crack propagation life for each type of sample where the abscissa is the number of cycles up to the flaking. The plots show the number of cycles when each type of crack was observed. Except Inclusion F in T-Type sample which was not cause the first flaking, the propagation life of the horizontal crack is almost similar for all types of sample because this process occured far away from the inclusion at their initiation site and the macroscopic crack propagation behavior may not be affected by the orientation of inclusions, then the difference of fatigue life between the orientation of inclusions should be attributed to the difference in the vertical crack initiation and the propagation lives. Then, the main difference in the flaking life for L-Type sample between other samples must originates from the crack initiation and the vertical crack propagation in the rolling direction.

\section{Conclusions}

In the present study, 4D observations of the formation and propagation of cracks in the rolling contact fatigue (RCF) tests were performed on a highstrength steel by combining a newly developed compact RCF testing machine with synchrotron radiation computed laminography (SRCL), and the crack propagation behavior and the fracture mechanism under RCF was discussed on specimens with MnS inclusions elongated in the rolling direction (L-Type). The following results were obtained.

(1) A vertical crack was initiated on the surface, parallel to the ball-rolling direction in specimens. The crack propagation direction was then changed perpendicular to the rolling direction. Thereafter, similar with S-Type or T-Type samples, vertical cracks then cause the horizontal cracks, parallel to the sample surface, when the vertical cracks reached a critical depth.

(2) The main difference for L-Type sample between other samples must originated from the crack initiation

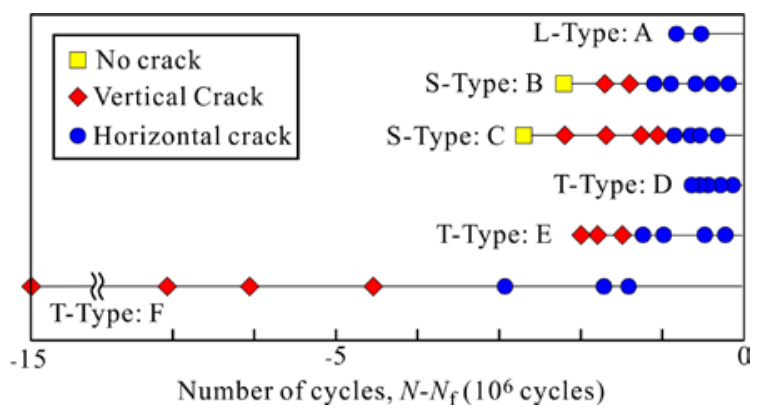

Figure 15. Number of ccyles for crack propagation. 
and propagation in the rolling direction. The fatigue life from the horizontal crack formation to the flaking is almost identical independent of the inclusion orientation.

\section{Acknowledgment}

The synchrotron radiation experiments were performed at beam line BL46XU of SPring-8 with the approval of the Japan Synchrotron Radiation Research Institute (JASRI) under proposal numbers 2017A1581, 2017B1610, and 2017B1908. The authors are grateful for the technical support of Dr. K. Kajiwara (JASRI).

\section{References}

1. L. Gustave, and A. Palmgren, J. App. Mech., Trans. ASME, 16, 165 (1949).

2. H. Styri, Proc.ASTM, 51, 682-700 (1951).

3. J.A. Martin, S.F. Borgese, and A.D. Eberhardt, J. Basic Eng., Trans. ASME, 88, 555-565 (1966).

4. K. Sugino, K. Miyamoto, M. Nagumo, and K. Aoko, Trans. ISIJ, 10, 98-111 (1970).

5. P.C. Becker, Metals Technology, 234-243 (1981).

6. A. Grabulov, U. Ziese, and H.W. Zandbergen, Scripta Materialia, 57, 635-638 (2007).

7. A. Grabulov, R. Petrov, and H.W. Zandbergen, Int. J. Fatigue, 32, 576-583 (2010).

8. M. Nagao, K. Hiraoka, and Y. Unigame, Sanyo Technical Report, 12: 38-45 (2005).

9. T. Tsuchida, and E. Tamura, Kobe Steel Engineering Reports, 61, 62-65 (2011).

10. A. Stiénon, A. Fazekas, and J.-Y. Buffière, A. Vincent, P. Daguier, F. Merchi, Materials Sci. Eng. A, 513-514: 376-383 (2009).
11. A. Stienon, A. Fazekas, J.-Y. Buffiere, P. Daguier, F. Merchi, and A. Vincent, J. ASTM Int., 7, 1-14 (2010).

12. D. Shiozawa., Y. Nakai., Y. Fukuda, Y. Neishi., and T. Makino., Proc. 15th Int. Conf. Exp. Mech., CD-ROM, No. 2635 (2012).

13. T. Makino., Y. Neishi, D. Shiozawa, Y. Fukuda, K. Kajiwara., and Y. Nakai, Int. J. Fatigue, 68, 168177 (2014).

14. D. Shiozawa., T. Makino., Y. Neishi., and Y. Nakai., Procedia Mat. Sci., 3, 159-164 (2014).

15. Y. Nakai, D. Shiozawa., S. Kikuchi., K. Sato, K., T. Obama, T. Makino., and Y. Neishi, Frattura ed Integrità Strutturale, 34, 267-275 (2014).

16. S. Gondrom, J. Zhou, M. Maisl, H. Reiter, M. Kroning, and W. Arnold, Nuclear Eng. Design, 190, 141-147 (1999).

17. Y. Nakai, D. Shiozawa, S. Kikuchi, T. Obama, H. Saito, T. Makino, and Y. Neishi, Eng. Fract. Mech., 183, 108-189 (2017).

18. Y. Nakai, D. Shiozawa, T. Kurimura, and K. Kajiwara, Proc. SPIE, 7522, 75224B: 1-7 (2010).

19. R. Adams, and L. Bischof, Analysis and Machine Intelligence, 16: 641 - 647 (1994).

20. T. Makino, Y. Neishi, D. Shiozawa., S. Kikuchi., T. Okada., K. Kajiwara., and Y. Nakai, Int. J. Fatigue, 92, 507-516 (2016).

21. S.P. Timoshenko and J.N. Goodier, Theory of Elasticity, Third Ed., 398 (McGraw-Hill Kogakusha, Ltd., Tokyo, 1970).

22. T. Makino, Y. Neishi, D. Shiozawa, S. Kikuchi, H. Saito, K. Kajiwara and Y. Nakai, Procedia Struct. Integ., $\quad 7, \quad 468-475$, (2017). 